\title{
COMMISSIONING MEASUREMENTS OF ELECTRON BEAM USING MONTECARLO ALGORITHM ON TRUEBEAM STX® AND COMPARIAON WITH CLINAC iX LINEAR ACCELERATOR
}

\author{
*Kamal SAIDI ${ }^{1,2}$, Redouane EL BAYDAOUI ${ }^{1}$, , Hanae EL GOUACH ${ }^{3}$, Othmane KAANOUCH ${ }^{1,2}$, \\ Mohamed Reda MESRADI ${ }^{1}$ \\ ${ }^{I}$ Hassan First University of Settat, High Institute of Health Sciences, Laboratory of Sciences and Health Technologies, BP \\ 555, 26000, Settat, Morocco \\ ${ }^{2}$ Department of Radiotherapy, Sheikh Khalifa International University Hospital, BP 82403, Casablanca, Morocco. \\ ${ }^{3}$ Hassan First University of Settat, FST, Laboratory of Analysis of Systems and Information Processing, B.P 577, 26000, \\ Settat, Morocco \\ *k.saidi@uhp.ac.ma
}

\begin{abstract}
TrueBeam STx latest generation linear accelerators (linacs) installed at Sheikh Khalifa International University Hospital in Casablanca, Morocco.

The aim of this is to present and compare the result of the Electron commissioning measurement on TrueBeam Stx and clinac iX installed at Sheikh Khalifa International University Hospital in Casablanca, Morocco. A compariaon of eMC calculations and measurements for TrueBeam Stx were evaluated.

Dosimetric parameters are systematically measured using a large water phantom 3D scanning system MP3 Water Phantom (PTW, Freiburg, Germany). The data of the electron beams commissioning including depth dose curves for each applicator, depth dose curves without applicator and the profile in air for a large field size 40x $40 \mathrm{~cm}^{2}$, and the Absolute Dose (cGy/MU) for each applicator. All the data were examined and compared for five electron beams (E6MeV, E9MeV, E12MeV, E16MeV and E20MeV) of Varian's TrueBeam STx and Clinac iX machines.

A comparison, between measurement PDDs and calculated by the Eclipse electron Monte Carlo (eMC) algorithm were performed to validate Truebeam Stx commissioning. All this measurements were performed with a Roos and Markus plane parallel chamber.
\end{abstract}


Our measured data indicated that electron beam PDDs from the TrueBeam Stx machine are well matched to those from our Varian Clinac iX machine.

Significant differences between TrueBeam and Clinac iX were found in in-air profiles and open field output. Maximum depth dose for the TrueBeam Stx and Clinac iX for the following energies $(6,9,12,16,20 \mathrm{MeV})$ are respectively $(1.15 ; 1.89 ; 2.6 ; 3.1$; and 2.35) and (1.24; $1.95 ; 2.70 ; 2.99$ and $2.4 \mathrm{~cm}$ ). For the TrueBeam Stx and Clinac iX the quality index $\mathrm{R}_{50}$ for applicator $15 \times 15 \mathrm{~cm}^{2}$ are in the tolerance intervals. Surface dose increases by increasing energy for both machines.

The Absolute Dose (cGy/MU) calibrated for both machine in $\mathrm{D}_{\max }$ at $1 \mathrm{cGy} / \mathrm{MU}$ for the reference field size cone $15 \times 15 \mathrm{~cm}^{2}$.

Bremsstrahlung tail Rp per energy levels as follows for the TrueBeam Stx : $6 \mathrm{MeV}-2.85 \mathrm{~cm}$, $9 \mathrm{MeV}-4.28 \mathrm{~cm}, 12 \mathrm{MeV}-5.97 \mathrm{~cm}, 16 \mathrm{MeV}-7.88 \mathrm{~cm}$ and $20 \mathrm{MeV}-9.86 \mathrm{~cm}$. and for the Clinac iX : $6 \mathrm{MeV}-2.86 \mathrm{~cm}, 9 \mathrm{MeV}-4.32 \mathrm{~cm}, 12 \mathrm{MeV}-5.96 \mathrm{~cm}, 16 \mathrm{MeV}-7.93 \mathrm{~cm}$ and $20 \mathrm{MeV}-10.08 \mathrm{~cm}$.

A good agreement between modeled and measured data is observed.

Keywords : TrueBeam STX®, Clinac iX, Electron Monte Carlo algorithm, Commissioning. 